le nord et sa perception des menaces émanant du sud: les retombées des crises maghrébines

\title{
Mustapha Sehimi
}

\section{(2) OpenEdition}

12 Journals

\section{Édition électronique}

URL : http://journals.openedition.org/conflits/95

DOI : $10.4000 /$ conflits.95

ISSN : $1777-5345$

Éditeur :

CCLS - Centre d'études sur les conflits lilberté et sécurité, L'Harmattan

Édition imprimée

Date de publication : 17 mai 1991

ISSN : 1157-996X

Référence électronique

Mustapha Sehimi, «le nord et sa perception des menaces émanant du sud: les retombées des crises maghrébines », Cultures \& Conflits [En ligne], 02 I printemps 1991, mis en ligne le 30 décembre 2002, consulté le 30 mars 2021. URL : http://journals.openedition.org/conflits/95 ; DOI : https://doi.org/ $10.4000 /$ conflits. 95

Ce document a été généré automatiquement le 30 mars 2021.

Creative Commons License 


\title{
le nord et sa perception des menaces émanant du sud: les retombées des crises maghrébines
}

\author{
Mustapha Sehimi
}

"Un missile mer-sol islamique sur Toulon est au moins aussi crédible qu'une division
aéroportée soviétique sur Hambourg. Quinze pays travaillent depuis 40 ans sur le
deuxième scénario, et tout est prêt pour le traiter convenablement. Le premier nous
prendrait au dépourvu..." Boutade que ce cas de figure de Régis Debray tout cas, le Sud
est de plus en plus perçu comme un danger. Un fantasme collectif est même entretenu
à cet égard par tel parti d'extrême droite, en France et ailleurs, sur la revanche de
Poitiers qui se dessine derrière les barbus islamistes d'Alger ou de Tunis même lorsque
le discours matois d'un Abbès El Madani prône le dialogue et la coopération avec
l'Occident. La guerre du Golfe a mis en exergue les clivages de la région: d'un côté, le
Nord dans le sillon de Washington paraissant mener une "croisade" et choqué par les
vociférations pro-irakiennes des masses maghrébines et arabes; de l'autre côté, celles-
ci trouvant là l'occasion de se ressourcer dans un nationalisme arabe que la vague
islamiste des dernières années avait quelque peu occulté. L'altérité Nord-Sud se
complique ainsi alors qu'elle avait été réduite à une dialectique simple Occident/Islam;
elle risque encore de s'aggraver avec l'état sinistré des économies du Sud confrontées
qu'elles sont à l'endettement, à l'explosion démographique, au chômage et à
l'augmentation des inégalités ; tout cela nourrit les appréhensions du Nord. Placé, chez
lui, devant les problèmes aigus d'intégration de millions d immigrés, il redoute
obscurément les retombées d'une crise politique, démographique et économique qui
sévit déjà de l'autre côté de la Méditerranée, et tout d'abord au Maghreb. L'insécurité
qui en résulte pourrait même, de son point de vue, ne pas être dépourvue d'une
dimension militaire. Tout indique qu'il est urgent d'y porter remède. Démocratie et islamisme

La scène politique maghrébine n'est plus ce qu'elle était voici deux ans seulement. L'Algérie de 1991, dont la dialectique interne a été débloquée par le choc des émeutes et de la répression d'octobre 1988 - après vingt-huit ans de "glacis" de parti unique - s'est 
engagée résolument dans un processus pouvant déboucher sur un système pluraliste. La Tunisie, elle, après le coup d'État "médico-légal" du 7 novembre 1987, destituant le président Bourguiba au profit de Zine El Abidine Ben Ali, est encore à la recherche d'une formule démocratique. Au Maroc, la probable tenue d'un référendum sur le Sahara permet d'espérer la relance et la consolidation de la démocratie. Même la Libye paraît avoir atténué fortement les ferveurs de la jamahirisation des années passées pour davantage de pragmatisme.

Institutions et sociétés maghrébines

Plus que d'aménagements institutionnels, il faut donc bien voir que l'on est face de changements dont la portée n'est pas encore épuisée. Par exemple, alors qu'il y a seulement une dizaine d'années encore, l'islamisme n'était qu'un extrémisme verbal marginal, tel n'est plus le cas aujourd'hui où il campe d'un bon pied dans l'aire araboislamique, se présentant même en Algérie, et en Tunisie surtout, comme la seule alternative. C'est que l'espace arabo-musulman ne peut rester à l'abri des spectaculaires mouvements de fond qui marquent l'Europe de l'Est mais aussi l'Afrique australe. Des évolutions considérables s'y préparent en effet; dans l'Afrique du Nord elles paraissent bel et bien engagées. Ce monde-là, le Sud, était naguère un enjeu politique et diplomatique de la guerre froide et de la rivalité Est-Ouest; avec le rapprochement américano-soviétique, il a perdu sa capacité de négociation. Avec la perestroïka, la nouvelle politique de Gorbatchev lui a fait perdre une bonne part de son importance dans les affaires du monde; il est marginalisé ; il se marginalise aussi. Le Maghreb, s'il continue à subir les effets des convulsions de la guerre du Golfe, n'en continue pas moins à tenter de faire face aux soubresauts des processus de démocratisation en cours. La problématique - pour l'Algérie et la Tunisie en tout cas est celle-ci : la démocratie ou l'islamisme? Faut-il jouer à fond la carte démocratique et prendre ainsi le risque de porter au pouvoir des forces islamistes considérées comme non-démocratiques? Après le totalitarisme des partis uniques (FLN algérien et néoDestour tunisien), faut-il n'avoir d'autre "sortie" que le totalitarisme islamique? D'autant plus que le FIS algérien met en avant "une alternative totale et globale", dans le cadre de l'Islam, aux grands problèmes politiques, idéologiques, économiques et sociaux. La Tunisie, elle-aussi, se trouve dans une impasse démocratique. Elle apparaît aujourd'hui à la traîne au regard du bouillonnement politique et médiatique marquant la vie du grand voisin algérien. Au Maroc, les difficultés sociales sont aiguës : chômage des jeunes, urbanisation accélérée, détérioration du pouvoir d'achat, etc. Mais le royaume "fait figure de pays stable dans le tourbillon des crises arabes"(2). Il reste que s'il n'y a pas de menace intégriste, comme chez ses voisins le roi étant chef religieux et couvrant ainsi tout le champ spirituel, la question des droits de l'homme empoisonne la vie politique locale et les relations avec Paris en particulier, mais aussi avec de nombreux pays occidentaux aiguillonnés par les organisations humanitaires. Quant à la Libye et à la Mauritanie, elles se sont laissées entraîner dans des conflits avec leur voisin du Sud (Tchad et Soudan pour la première, Sénégal pour la seconde), réveillant ainsi l'irrédentisme arabe et africain

En France, l'inquiétude islamique

L'Islam peut-il être "tranquille" ? Le danger d'un Islam pur et dur - surtout en Algérie avec le succès du FIS aux élections locales de juin 1990 - ne menace pas que la stabilité et la sécurité au Maghreb et en Méditerranée occidentale même : il compromet aussi la politique d'immigration de l'Europe ainsi que les chances d'intégration que se propose en particulier de mener la France. Ses appréhensions se nourrissent d'au moins deux 
données : les musulmans sont près de trois millions en France et plus de quatre millions et demi dans l'Europe communautaire. Mais c'est surtout dans l'Hexagone que la communauté musulmane s'est régulièrement développée et diversifiée. En effet, dans une première catégorie, l'on compte plus de un million de personnes de nationalité française se réclamant de l'Islam; elles sont réparties en trois groupes sociologiques : les 400000 Français-musulmans originaires d'Algérie et rapatriés en 1962 - ils sont particulièrement défavorisés et peu intégrés; les enfants d'immigrés de religion musulmane, appelés les Beurs, et qui sont pratiquement de la même taille - ils ont des rapports complexes avec leurs parents et témoignent de plus d'esprit revendicatif que leurs aînés parce que n'ayant pas d'autre option que... l'intégration! Enfin, les Français convertis à l'islam qui dépasseraient les 200000 personnes. Ce dernier groupe pose évidemment le moins de problèmes d'intégration puisqu'ils étaient au départ assimilés dans la société française.

On notera, soit dit en passant, que toutes ces données montrent bien que l'Islam en France est pluriethnique, une approche courante le réduisant pour des raisons souvent politiciennes de propagande à la seule communauté maghrébine. A côté de cette communauté de nationalité française, il y a lieu de dénombrer plus de 1,8 million de musulmans de nationalité étrangère répartis comme suit : 800000 Algériens, 550000 Marocains, 250000 tunisiens, 120000 Turcs et 100000 musulmans d'Afrique noire (surtout du Mali et du Sénégal), ainsi que quelques milliers d'autres de diverses origines. Ces chiffres attestent d'une réalité : l'islam est la deuxième religion des français. Ce qui pose la question délicate, et souvent passionnée, de la place de cette religion dans la structure actuelle de la société française. Autrement dit: de quelle manière se fera la politique d'intégration? Il faut bien dire ici que la forte poussée intégriste en Algérie rejaillit au coeur même du problème brûlant de l'intégration. Car c'est avec les Algériens, surtout, que les questions les plus épineuses se posent: mariages mixtes, double nationalité, droit de garde des enfants des couples séparés, ... C'est aussi avec eux que la société française rencontre le plus de problèmes, ne serait-ce que par suite du contentieux émotionnel lié à la guerre d'Algérie et aux conditions historiques particulières qui ont conduit à la décolonisation de ce pays. De plus, l'intégrisme en Algérie et ses diverses manifestations posent des interrogations de principe qui viennent d'ailleurs compliquer le débat franco-français sur l'intégration des immigrés. Que l'Algérie penche vers cet Islam intégriste ne peut que faire peser les pires menaces sur l'ensemble de la politique d'immigration et/ou d'intégration. Pourquoi ? Parce que malgré l'arrêt officiel des flux migratoires en 1974, les frontières françaises sont loin d'être étanches. Par le biais du regroupement familial, de l'immigration clandestine et de l'usage -jugé abusif du statut de réfugié politique, les flux d'entrée gardent encore un solde positif évalué tout de même à 100000 personnes par an en moyenne, dont la moitié au moins sont d'origine maghrébine.

Les autorités françaises se proposent de stopper ces flux d'entrée et la célèbre formule de M. Michel Rocard est significative de cet état d'esprit: "La France ne peut plus accueillir toute la misère du monde". Or, le mouvement d'émigration vers la France continue : en 1989, plus de 2000 avocats, juristes, médecins et ingénieurs ont quitté l'Algérie pour la France ; et l'on compte plus de professeurs maghrébins dans les lycées français, surtout dans les disciplines scientifiques, que l'ensemble des coopérants français au Maghreb. Sans doute, Paris laisse-t-il plutôt faire s'agissant d'une élite ; mais les craintes existent parmi certains responsables français que ce nouveau flux ne constitue l'avant-garde d'un courant migratoire renouvelé. Ainsi, M. François Léotard, 
lors d'une tournée en Algérie en février 1991, avait été saisi par le fait que les consulats français délivraient 2000 visas par jour, et pas seulement à des touristes... Si la fièvre islamique continue de monter outre Méditerranée, la France n'aura-t-elle pas d'autre choix que de fermer pratiquement ses frontières? Le pari actuel est de promouvoir un islam à la française, débarrassé de ses outrances, occidentalisé en somme, laïcisé pour tout dire. Mais l'on se trouve placé devant une difficulté de belle taille à cet égard: comment cerner l'organisation de cette religion si l'on sait que l'islam sunnite - qui est la grande majorité des Musulmans en France et en Europe - s'affirme, lui, sans clergé et sans organisation interne. Sans doute, le monde musulman n'est-il pas totalement inorganisé de ce côté-ci de la Méditerranée; mais il faut bien convenir que ses structures sont peu "lisibles". Un monde donc qui se veut unitaire mais qui, de fait est éclaté, multiple, flou : éclaté suivant les origines ethniques et nationales; multiple en ce sens que les rapports de cette communauté à l'islam sont différenciés ; flou encore car les champs propres au culturel, au politique et au religieux sont souvent entremêlés. Ce qui est souvent perçu d'ailleurs, c'est surtout un certain islam, extrémiste, intégriste, objet d'une surmédiatisation alors qu'il ne concerne qu'une petite partie de la communauté musulmane. Or, la grande majorité de celle-ci prône et pratique un "islam tranquille". D'ailleurs, l'idée de promotion de cet islam là et de son ancrage à la société française fait son chemin. Avec la mise en place de conseils islamiques départementaux officiels pour éviter tout caractère souterrain et incontrôlable ; avec aussi le projet de création d'une université théologique islamique à Strasbourg; sans oublier, à terme, le nécessaire rapprochement des valeurs islamiques avec le code civil et, dans le même temps, leur prise en compte par le droit positif français. Autant de mesures et d'initiatives qui convergent vers le même sens : celui d'un gallicanisme musulman dont la France pourrait être le Saint-Siège. S'il y a longtemps que la France n'est plus fille aînée de l'Église catholique, elle est désormais, volens nolens, marraine de trois millions de musulmans...

La peur de l'immigration et ses raisons

\section{- Déséquilibres démographiques et contraste économique}

Résumés à l'essentiel, les discours sur ces déséquilibres peuvent souvent exprimer ainsi. Une décélération rapide de la démographie des pays du Nord et une accélération exponentielle de celle des pays du Sud vont transformer radicalement les rapports autour de la Méditerranée.

- La peur de l'immigration

Ainsi, entre 1950 et 2025, la part des pays européens dans la population méditerranéenne sera pratique ment inversée par rapport à celle des pays de la rive africaine et asiatique chutant de 70 à $30 \%$ du total. Il s'ensuit que la pression démographique du Sud sera d'autant plus forte que la proportion des jeunes est beaucoup plus élevée dans les pays du Sud que dans ceux du Nord. Mais, des éléments objectifs nuancent les "angoisses" qui nourrissent l'idée d'une invasion des rives nord de la Méditerranée par celles du Sud. Pour ce qui est de la croissance de la population, tout d'abord, son rythme commence à se ralentir dès les années quatre-vingt dans le Sud, sauf en Algérie où cette tendance ne s'amorcera qu'à compter des années quatrevingt dix (Voir tableau à la fin de l'article). On observe, par ailleurs, qu'à la fin de la période de projection, vers 2020-25, les taux de natalité auront diminué de moitié environ. $S^{\prime}$ ils seront inférieurs à 20 pour mille, et seront comparativement identique à la situation des pays développés au début des années soixante-dix (c'est-à-dire pour un 
même taux de natalité), il n'en plus élevé du fait d'une mortalité très faible'. Cela dit, c'est. aujourd'hui que l'écart entre les évolutions démographiques de part et d'autre de la Méditerranée est le plus élevé : dans les quatre pays d'Afrique du Nord (Algérie, Egypte, Maroc, Tunisie), la croissance annuelle de la population est de $2,5 \%$ en moyenne alors qu'elle n'est que de $0,2 \%$ en Europe occidentale. Cet écart n'a pas cessé d'ailleurs d'augmenter depuis les années cinquante ; mais il va se réduire dès 1990-91: en effet, si la croissance de la population européenne va devenir de plus en plus faible jusqu'à la décroissance?-, autre côté le rythme de croissance de la population d'Afrique du Nord va diminuer encore plus rapidement. Dans ces conditions, il est vrai que le rapport numérique des populations du Nord et du Sud de la Méditerranée ne cesse de se rééquilibrer et s'inversera sans doute mais pas jusqu à "l'invasion" (tableau contraste Europe Afrique du nord (idem). Le problème tient autant, si problème il y a, au contraste des structures de population qu'à celui de la croissance démographique. En Afrique du Nord, par suite de la baisse de la natalité, la proportion des classes d'âge 15-65 ans va croître, mais l'âge médian qui partage la population en deux parties égales restera cependant assez bas. Dans le même temps, en Europe, cet âge médian grimpera de 33,9 à 40,8 ans ; en Allemagne, il atteindra même en 202545,9 ans pour 36,7 ans en 1985. Ce vieillissement est dû au gonflement des classes d'âges au-dessus de 65 ans. Ce vieillissement européen est tout autant relatif qu'absolu : en effet, entre 1985 et 2025, l'ensemble de la population européenne va augmenter de 10 mi11ions d'habitants mais le nombre des moins de 15 ans comme celui des 15-64 ans va diminuer; d'un autre côté, la population de plus de 64 ans va augmenter de 25 millions de personnes. Tableau, donc, au Nord de la Méditerranée, d'une société vieillissante qui est l'un des gros problèmes des temps à venir. En face dans l'Afrique du Nord, c'est l'irruption des classes d'âges jeunes qui est caractéristique de l'évolution démographique dans les trente années qui viennent. Le ratio de dépendance - c'est le rapport du nombre des moins de 15 ans et des plus de 65 ans à la population entre 15 et 65 ans- va, lui, fortement diminuer. Massivement, de jeunes générations au sud de la Méditerranée vont arriver sur le marché du travail. A n'en pas douter, ce contraste pèsera de tout son poids dans l'espace régional : qu'on le veuille ou non, la composante démographique sera décisive dans la structuration et l'évolution des rapports économiques et politiques entre les deux rives de la Méditerranée (Tableau sur l'évolution des structures par âge). Ces jeunes générations pourront-elles trouver à s'employer chez elles, ou seront-elles tentées par l'émigration? Ceci dépendra du développement des économies au sud de la Méditerranée.

La dérive des économies maghrébines

Après des hauts et beaucoup de bas dans leur politique de développement depuis les années soixante, des hypothèques majeures pèsent désormais sur les économies du Sud, maghrébines en particulier. La première hypothèque tient à ce qu'elles sont confrontées à la baisse tendancielle, depuis une dizaine d'années environ, du prix des matières premières - pétrole pour l'Algérie, la Libye et la Tunisie, phosphates pour le Maroc et la Tunisie, fer pour la Mauritanie, gaz naturel pour l'Algérie... Une autre contrainte regarde le poids de l'endettement, devenu franchement alarmant : en 1990, la dette dépassait les soixante milliards de dollars pour les cinq pays de l'Union du Maghreb Arabe (UMA), soit pratiquement un millier de dollars par habitant. Pareille situation est d'autant plus préoccupante qu'une partie significative des recettes d'exportation des pays maghrébins est affectée au paiement des importations alimentaires. Ainsi, au cours des dix dernières années, l'Algérie a importé, bon an mal 
an, plus de deux milliards de dollars de produits alimentaires, le Maroc pour huit cents millions de dollars et la Tunisie pour quatre cent cinquante millions de dollars. Cette dépendance alimentaire est la conséquence à la fois de causes externes baisse des prix mondiaux des produits agricoles qui compromet la compétitivité des agricultures du Sud face à celles du Nord-, mais aussi d'options de développement interne pour le moins discutables - priorité à l'industrie en Algérie et en Libye au détriment du secteur agricole - sans oublier une mauvaise appréciation des rapports cultures vivrières/ cultures spéculatives au Maroc et en Tunisie par suite de choix trop agroexportateurs(4). Sans doute, des correctifs ont été apportés ces dernières années dans le sens de la promotion de la production agricole; mais, à court terme, l'hypothèse d'une autosuffisance alimentaire n'est aucunement retenue, loin de là. De nombreux facteurs obèrent le développement du monde rural : limites liées au milieu naturel5, agraires(5); sans oublier que ces obstacles n'au raient certainement pas pesé si lourd si les politiques suivies dans ce domaine n'étaient pas venues aggraver un contexte naturel et social peu favorable au départ. Le déficit céréalier est symptomatique de cette dépendance. Aujourd'hui, dans les quatre pays d'Afrique du Nord (Algérie, Egypte, Maroc, Tunisie), la consommation repose en grande partie sur les importations, faute d'appui gouvernemental et des bas prix internationaux. D'autres déséquilibres sont aussi à prendre en considération : ils limitent singulièrement les chances de développement des pays du Maghreb. Ainsi, la densité de la population (10,6 hab/km2) est dix fois moindre qu'en France (103,1 hab/km2); mais l'exode rural sans cesse croissant et l'impasse faite sur l'espace saharien ( $80 \%$ de la superficie du Maghreb) au profit du littoral ont provoqué une forte saturation des bandes côtières et une concentration urbaine dramatique. Il y a basculement de la population vers le monde urbain au détriment du monde rural : la population urbaine dépasse (Tunisie) ou frôle (Algérie, Maroc) les 50 \%. Comme on l'a justement relevé, "les villes du Maghreb ont commencé à devenir de véritables mégapoles, en tout premier lieu Casablanca et Alger, dont les infrastructures de communication et d'assainissement sont engorgées et sécrètent un mal urbain parfois insupportable: pénuries d'eau à Alger, collapsus de la circulation automobile, sous-développement des transports publics et des télécommunications, prolifération des bidonvilles à Casablanca, Alger, Tunis, ...

Cette concentration urbaine vide les centres et les suds de leur population, livre des régions écologiques fragiles au sable, amène le désert aux portes des villes et crée de dangereux déséquilibres sur des bandes côtières surpeuplées"(7). Mais il y a plus, l'environnement va se dégrader dans des proportions inquiétantes et le problème de l'eau sera crucial... A telle enseigne que la survie économique dépend des solutions apportées aux problèmes de la gestion de l'eau, de celle des déchets, de la pollution marine et de la gestion du littoral. Les prévisions les plus pessimistes sont faites actuellement quant à la possibilité de résorption du chômage. La réduction de ce chômage en Algérie à l'horizon 2010 nécessiterait une croissance de 5,2 \% par an dans les secteurs non agricoles et non administratifs. Comme l'a relevé un économiste "du fait de la structure par âge de la population, le Maghreb aura à créer plus de six millions d'emplois durant la décennie 1990-2000 soit la moitié de sa population active actuelle..."(8). D où, inévitablement, s'il n'y a pas un réel développement économique dans les prochaines années - et plus encore à long terme - de formidables poussées dans le sens de l'accroissement des flux migratoires vers l'Europe. Comme l'a souligné Alfred Sauvy, "si les richesses ne vont pas là où sont les hommes, les hommes vont 
naturellement là où sont les richesses". Les experts pronostiquent donc des vagues migratoires Sud/Nord d'une grande ampleur. Ainsi Jacques Lesourne(9) estime qu'à l'horizon 2025, les migrants, ou leurs descendants, pourraient représenter en Europe 25 millions d'habitants dont vingt millions de musulmans. Il y a là des perspectives incontournables nourries et confortées par le fait que le sous-emploi au Sud est l'un des problèmes majeurs de la stabilité et de la sécurité que les uns et les autres appellent officiellement de leurs voeux. Ainsi la peur d'une immigration massive, même si elle est souvent fantasmatique, n'est dons pas dénuée de tout fondement. Son actualisation dépendra de la réussite ou de l'échec d'un co-développement permettant de résoudre sur place les problèmes de l'emploi. La peur de l'immigration n'est qu'un des deux vecteurs par lequel le Nord perçoit le Sud, le second est celui qui ouvrait notre article et que la guerre du Golfe a actualisé, le risque d'un affrontement militaire entre les deux rives de la Méditerranée.

Le risque d'affrontement militaire?

Surarmement et militarisation du Maghreb: le bilan polémologique de la zone maghrébine, sans parler du Machrek où les problèmes de paix et de sécurité sont d'une autre nature et d'une autre dimension, fait apparaître quelque trente ans après les indépendances, un ensemble de tensions, de conflits latents ou larvés; pendant longtemps, tout cela ne pouvait faire régner au Maghreb qu'un climat de discorde et d'hostilité, souvent mal contenues d'ailleurs. L'altérité entre les régimes sociopolitiques et les politiques économiques, les positionnements différenciés dans le système international ainsi que la persistance de contentieux frontaliers ne pouvaient que peser sur la nature des relations inter-maghrébines. Tous ces éléments expliquent que des contraintes particulières aient joué dans le sens de politiques de défense très actives. On observera pour commencer qu'aucun des pays de l'UMA n'a échappé à ce phénomène très contemporain: l'accroissement global de l'effort militaire. La persistance d'un climat de guerre froide, de tension et même de conflit (Sahara, "guerre des sables" maroco-algérienne d'octobre 1963, agression libyenne contre Gafsa en janvier $1980, \ldots)$, jusqu'à ces toutes ces dernières années en tout cas, a poussé dans ce sens; la forte augmentation des revenus pétroliers après 1973, en particulier pour ce qui est de l'Algérie et de la Libye, explique aussi cette tendance; mais il ne faut pas non plus omettre que le renforcement de l'appareil militaire répondait également au souci des différents dirigeants maghrébins de défendre et de conforter leurs régimes respectifs, chacun d'entre eux suivant une problématique de légitimation propre. Ainsi, depuis une douzaine d'années au moins, les dépenses militaires de chacun de pays maghrébins ont été en effet importantes et traduisent une tendance à la hausse (cf. tableau sur évolution des dépenses militaires 1973 1990).

De ces chiffres, on peut tirer un certain nombre d'indications significatives. La première d'entre elles est la part importante des dépenses militaires dans le PIB - c'est particulièrement vrai pour la Libye (12\%) mais aussi pour le Maroc $(5,2 \%)$ et la Tunisie (5,6\%). Les dépenses de défense de l'Égypte frappent elles aussi par leur importance: déjà élevées en 1988 (5,73 milliards), elles ont grimpé à 6,81 milliards de dollars en 1989 pour se situer encore à hauteur de 6,38 milliards de dollars en 1990. Une autre remarque a trait aux montants très importants des dépenses militaires qui contrastent de façon saisissante avec la modicité des dépenses sociales (éducation mais surtout santé). Le budget militaire tunisien est typique du renforcement accordé à la place et au rôle des armées dans les systèmes politiques de la région : il a en effet pratiquement triplé entre 1982 et 1986. Sur les dix dernières années, ces dépenses présentent un 
profil très heurté : elles flambent en 1979, décroissent un peu ensuite, pour augmenter à nouveau en 1982-83. Cette volatilité a une double explication: elle est liée, non seulement aux émeutes populaires de 1978 et 1981, mais aussi à l'agression menée contre Gafsa (janvier 1980) par Tripoli et aux menaces que fait peser ainsi Kadhafi sur ce pays. Les dépenses militaires marocaines et algériennes se maintiennent également à un haut ni veau, en particulier, en raison du conflit du Sahara et des menaces, réelles ou prospectives, qui y sont liées. Cette "militarisation" des budgets s'est naturellement accompagnée d'un gonflement substantiel des effectifs militaires dans tous les pays maghrébins et arabes (tableau sur évolution des forces armées 1973 1990). L'Afrique du Nord reste encore un théâtre de conflits (Tchad, Sahara, Soudan, etc.). Simultanément, les conflits du Proche-Orient ont soit débordé sur le Maghreb(10) soit fortement agité les relations interarabes. L'apaisement observé entre 1988-90 au Maghreb en particulier et qui a conduit à la création de l'UMA n'a fait tache d'huile que d'une manière éphémère au Moyen-Orient avec la fin de la guerre Irak-Iran. La guerre du Golfe et ses conséquences en 1990-91 ne peuvent qu'aviver les perceptions de menaces dans tout l'ensemble Maghreb-Machrek.

Menaces prospectives et formes de conflictualité

Si le Sud se sent davantage menacé aujourd'hui avec "l'impérialisme" américanooccidental qui s'est manifesté lors de la guerre du Golfe, force est de dire aussi que pour le Nord, le Sud n'est pas non plus sécurisant. Des menaces réelles ou prospectives existent à cet égard; elles expriment des formes de conflictualité particulières. On distinguera ici entre les menaces pesant sur la sécurité et celles relatives à la stabilité : les unes et les autres se différenciant par la gravité de leur contenu. En ce qui concerne la première catégorie, il faut bien souligner que la plus grande menace est sans conteste le recours ou vert à la force armée entre deux ou plusieurs pays de la région. L'annexion du Koweït par l'Irak, le 2 août 1990, est sans doute un cas de figure extrême; mais l'histoire des vingt ou trente dernières années au Maghreb et au Machrek, et plus globalement encore au Sud, atteste que les incidents armés et les conflits localisés sont plus fréquents qu'il n'y paraît. Tout s'y passe comme si la prégnance d'une forme particulière d'une culture des conflits était une norme bien supérieure à la préservation de la paix et au respect du droit international. Les guerres civiles restent un spectre encore réel; l'instabilité politique prédomine; l'armée n'est pas à l'abri des secousses pouvant ébranler les systèmes politiques en place; et puis la violence urbaine s'installe, de Casablanca à Alger, de Tunis au Caire - les dysfonctionnements des cadres institutionnels existants ont fait que, depuis une bonne dizaine d'années au moins, les "émeutes du pain" participent désormais de plain-pied à la régulation des crises. La prolifération des armes chimiques, bactériologiques et de destruction de masse constitue un autre danger.

La guerre du Golfe a montré la portée de ce phénomène. Elle a aussi montré que s'il y avait bien une barrière stratégique formée par la Méditerranée entre le Nord et le Sud, il n'en restait pas moins une grande proximité, surtout pour des missiles entre l'Espagne et le Maroc ou encore entre l'Italie et la Tunisie. S'agissant, en second lieu, des menaces pesant sur la stabilité de la zone, c'est une belle palette qu'il faut mettre en exergue: prise de pouvoir par des régimes islamistes jugés xénophobes, antioccidentaux; établissement de régimes militaristes dans une multitude de combinaisons avec les précédents; guerres civiles internes; terrorisme; exode migratoire, ... Dans tous ces cas de figure, la violence s'installe conduisant à porter atteinte aux biens et aux personnes des pays de la région, européens surtout. D'autant 
que ces formes de violence peuvent fort bien ne pas s'exprimer d'une manière isolée mais dans une dialectique synergique entre les unes et les autres. Si le déséquilibre des forces militaires entre le Nord et le Sud s'accompagne pour l'heure d'une dissuasion décisive du premier sur le second, des explosions de violence ne sont pas pour autant à exclure, les pays du Sud pouvant opter pour des actions ponctuelles dans ce domaine. Les principaux points d'accrochage concernent les frontières terrestres en Afrique entre l'Espagne et le Maroc (Ceuta et Mellilia) mais aussi les côtes italiennes; des incidents pourraient être provoqués par des actions sur des bateaux et des aéronefs en transit dans les espaces communs de la Méditerranée. Enfin, les tensions des relations Sud-Sud ne sont pas les moins préoccupantes qu'il s'agisse du conflit du Sahara (MarocAlgérie) ou des différends entre la Tunisie et la Libye, ou même encore entre Tripoli et Le Caire(11).

Une nouvelle sécurité ?

Le risque d'affrontement militaire direct et prolongé est donc négligeable, étant donné la disproportion du rapport de for ces mais les incidences sur le Nord de conflits au sein du Maghreb ou d'exaspérations momentanées sont crédibles. Aussi faut-il y pallier dès maintenant en mettant en place une nouvelle sécurité. Que faire alors? N'y-a-t-il pas lieu au moment où l'on nous parle tant d'un nouvel ordre international - confondu ici ou là avec les Etats-Unis se présentant volontiers comme les gendarmes de la sécurité mondiale - de se pencher sur les conditions et les modalités de promotion d'un espace stabilisé dans la région? A n'en pas douter, ce qui fait défaut - au Maghreb, au Machrek (la crise du Golfe en a été l'illustration tragique) et dans le Sud -, c'est un système de prévention des crises. Mais comment organiser cette sécurité collective? En Méditerranée, l'idée d'une conférence sur la sécurité et la coopération (CSCM) sur le modèle, mutatis mutandis, de la CSCE d'Helsinki, fait son chemin. Un projet plus limité, borné aux riverains de la Méditerranée occidentale (les quatre de la rive Nord Portugal, Espagne, France, Italie- et les cinq de l'UMA -Mauritanie, Maroc, Algérie, Tunisie, Libye, avec Malte) circule dans les chancelleries. Il n'est pas sûr qu'il puisse se concrétiser tant la coupure a été totale entre le Nord et le Sud lors de la guerre du Golfe, les Maghrébins jugeant en particulier que Paris s'était pratiquement alignée sur Washington, malgré sa politique arabe. Qu'une reprise du dialogue et de la concertation entre la France et le Maghreb, et plus globalement entre la CEE et l'UMA, soit ces temps-ci inscrite à l'ordre du jour, voilà qui est significatif de l'état d'esprit de l'après-Golfe; mais il reste à aller au-delà de la normalisation des relations interétatiques et à surmonter les contentieux émotionnels entre les peuples que la guerre du Golfe n'a fait que raviver. Une autre direction pourrait être la protection d'un "espace juridique commun"(12) où l'espace de légalité serait la préoccupation prioritaire; où la coopération régionale se mobiliserait contre des maux tels que la contrebande, le trafic de drogue, les trafics de main-d'oeuvre, la lutte antiterroriste(13); où l'espace maritime et aérien serait clairement assigné à la protection de l'environnement et la préservation des ressources halieutiques fonderaient une nouvelle solidarité régionale; et où enfin toutes les conditions seraient réunies pour faciliter la promotion d'une zone de codéveloppement évitant l'intensification des flux migratoires. Vaste programme, on le voit bien, dont l'ambition serait, en s'attaquant aux véritables problèmes, de ruiner la construction idéologique sur la menace que fait peser l'Autre de part et d'autre de la Méditerranée. Lors du sommet des neuf pays riverains de la Méditerranée, à Rome, le 10 octobre 1990, les ministres des Affaires étrangères ont souligné que "le dialogue, la concertation et la coopération" s'impose pour identifier, définir et résoudre les 
problèmes de sécurité d'intérêt commun dans la région; de même, ils ont mis l'accent sur le fait que cette dynamique devait revêtir "un caractère global, intégrant les paramètres politiques, de sécurité, économiques, culturels, humains et écologiques". II reste à aller au-delà de cette profession de foi et à la traduire en termes institutionnels, politiques et diplomatiques conséquents.

Pourcentage des moins de 15 ans dans la population totale

Europe du Sud :

(Portugal, Espagne, France, Italie, Yougoslavie, Albanie, Grèce, Malte)

$1950: 26,41980: 23,52000: 19,72025: 18,4$

Afrique :

(Égypte, Libye, Algérie, Maroc)

$1950: 41,71980: 432000: 38,32025: 27,7$

(Source : doc. original prof Raimondo Cagiano de Azevedo, université de Rome)

Évolution des dépenses militaires 1973-1990 (en millions de dollars 197319761982 198619891990 \% du PIB

Algérie 213589848116185010102

Libye 19336470913901420103912 (est.)

Maroc 21368413281136121613605.2

Tunisie 39681195204833885.6

\section{RÉSUMÉS}

La Guerre du Golfe a mis en évidence les intérêts contradictoires entre les peuples du Nord et ceux du Maghreb. Confronté à l'intégration de milliers d'immigrants, le Nord craint les conséquences d'une crise se poursuivant au Maghreb. L'auteur analyse la réalité objective se cachant derrière ces menaces. Si le danger d'une immigration massive Sud/Nord n'est pas dénué de tout fondement, son ampleur dépendra beaucoup de l'issue favorable ou de l'échec éventuel d'un développement concentré ou code de développement. Quant au risque d'un conflit armé direct et étendu, il reste hautement hypothétique, lorsque l'on considère les forces en présence. Cependant, les incidence, sur le Nord, de l'incertitude régnant actuellement au Maghreb, doivent être prises en compte. Pour conclure, l'auteur analyse les conditions de l'établissement d'une nouvelle sécurité autour d'une possible sphère juridique commune afin de prévenir d'autres crises.

The Gulf War made obvious the opposing interests confronting the North to the Maghreb's masses. Facing the integration of thousands of North African immigrants, the North fears the consequences of a continuing crisis in the Maghreb. The author tries to evaluate the objective reality behind these threats : if the danger of a massive South/North migration is not unfounded, its scale will depend much upon the success or eventual failure of a codevelopment programme. As for the risk of a direct and extended armed conflict, it remains highly hypothetical if we consider the disproportion between the opposing military forces. However, the outcomes in the North of the present uncertainty in Maghreb must not be ignored. To make an inference, the 
author analyses conditions for the creation of a new security pact organised to prevent the occurrence of new crises around a possible new common judicial sphere.

INDEX

Mots-clés : menace, pauvreté, discours sécuritaire

Index géographique : Maghreb, méditerranée, Pays du Sud (Tiers Monde)

\section{AUTEUR}

\section{MUSTAPHA SEHIMI}

Professeur de droit public à l'Université Mohammed V, directeur de recherche au Centre d'Etudes Stratégiques de Rabat 